\title{
Interaction of the quantized electromagnetic field with atoms in the presence of dispersing and absorbing dielectric bodies
}

\author{
S. Scheel and D.-G. Welsch \\ Theoretisch-Physikalisches Institut, \\ Friedrich-Schiller-Universität Jena, \\ Max-Wien-Platz 1, D-07743 Jena, Germany
}

\begin{abstract}
A general theory of the interaction of the quantized electromagnetic field with atoms in the presence of dispersing and absorbing dielectric bodies of given Kramers-Kronig consistent permittivities is developed. It is based on a source-quantity representation of the electromagnetic field, in which the electromagnetic-field operators are expressed in terms of a continuous set of fundamental bosonic fields via the Green tensor of the classical problem. Introducing scalar and vector potentials, the formalism is extended in order to include in the theory the interaction of the quantized electromagnetic field with additional atoms. Both the minimal-coupling scheme and the multipolarcoupling scheme are considered. The theory replaces the standard concept of mode decomposition which fails for complex permittivities. It enables us to treat the effects of dispersion and absorption in a consistent way and to give a unified approach to the atom-field interaction, without any restriction to a particular interaction regime in a particular frequency range. All relevant information about the dielectric bodies such as form and intrinsic dispersion and absorption is contained in the Green tensor. The application of the theory to the spontaneous decay of an excited atom in the presence of dispersing and absorbing bodies is addressed.
\end{abstract}

\section{Introduction}

Optical instruments such as beam splitters or cavities are more or less complicated macroscopic material bodies, whose use in experiments requires careful examination with regard to their action on the light under study. In quantum optics an important consideration is the influence of the presence of material bodies on the quantum statistics of the light. It is therefore necessary to take account of the presence of material bodies when considering the interaction of quantized light with atomic systems. In principle, such bodies could be included as a part of the matter to which the radiation field is coupled and treated microscopically. However there is a class of 
material bodies whose action can be included in the quantum theory exactly, namely dielectric bodies that respond linearly to the electromagnetic field.

The quantum theory of radiation in the presence of dielectric media has been studied over a long period. Commonly, dielectric matter is characterized by the permittivity, which describes the response of the matter to the electric field. Quantization of the electromagnetic field in dielectrics with real and frequency-independent permittivity has been treated extensively [1, 2, 3, 4, 5, 6, 0, 8, 9, 10, 11, 12, 13. In the same context, dispersive dielectrics have been considered [14, 15, 16, 17, 18, 19, 20. However, it is well known that the permittivity is a complex function of frequency which has to satisfy the Kramers-Kronig relations which state that the real part of the permittivity (responsible for dispersion) and the imaginary part (responsible for absorption) are necessarily connected with each other. Hence, any quantum theory that is based on the assumption of a real permittivity can only be valid for narrow-bandwidth fields far from medium resonances where absorption can safely be disregarded.

A systematic and quantum-theoretically consistent approach to the problem was developed by Huttner and Barnett [21] on the basis of the microscopic Hopfield model of a bulk dielectric [22]. They performed an explicit Fano-type diagonalization [23] of a Hamiltonian consisting of the electromagnetic field, a (harmonic-oscillator) polarization field representing the dielectric matter, and a continuous set of (harmonicoscillator) reservoir variables accounting for absorption. The resulting expression for the vector potential could also be written in terms of the Green function of the classical scattering problem as was realized by Gruner and Welsch 24, 25]. That, in fact, makes it possible to perform the quantization of the electromagnetic field in the presence of arbitrary dielectric bodies of phenomenologically given KramersKronig consistent permittivities, without referring to specific microscopic models of the bodies, which are hard to establish for general systems [26, 27.

Having quantized the electromagnetic field, the question arises of how to include in the theory the interaction of the medium-assisted field with atomic systems in order to study the influence of dielectric bodies on quantum-optical processes such as spontaneous decay of an excited atom near material bodies or in micro-cavities. It is well known that the spontaneous decay can be strongly modified when the atom is placed inside a high-quality micro-cavity. Recent progress in constructing microcavities has rendered it possible to approach the ultimate quality level determined by intrinsic material losses, so that the question of the influence of absorbing material has been of increasing interest. In what follows we develop a basic-theoretical concept for treating the interaction of the electromagnetic field with atomic systems in the presence of dielectric bodies of (phenomenologically) given arbitrary complex permittivities.

The paper is organized as follows. In Sec. 2 the quantization of the electromagnetic field on a dielectric background of complex permittivity is outlined. The interaction of the medium-assisted electromagnetic field with atomic systems is studied in Sec. 3 and the minimal-coupling and multipolar-coupling Hamiltonians are derived. Finally, 
a summary and some concluding remarks are given in Sec. 因.

\section{Quantization of the electromagnetic field in the presence of dielectric media}

As already mentioned, dielectric matter plays an important role in optics, because (passive) optical instruments are typically composed of dielectrics. In classical electrodynamics, dielectric matter is commonly described in terms of a phenomenologically introduced dielectric susceptibility (or permittivity). This concept has the benefit of being universally valid, because it uses only general physical properties, without the need of involved $a b$ initio calculations.

\subsection{Classical basic equations}

The phenomenological Maxwell equations of the electromagnetic field in the presence of dielectric bodies but without additional charge and current densities read

$$
\begin{gathered}
\boldsymbol{\nabla} \cdot \mathbf{B}(\mathbf{r})=0, \\
\nabla \times \mathbf{E}(\mathbf{r})+\dot{\mathbf{B}}(\mathbf{r})=0, \\
\boldsymbol{\nabla} \cdot \mathbf{D}(\mathbf{r})=0, \\
\nabla \times \mathbf{H}(\mathbf{r})-\dot{\mathbf{D}}(\mathbf{r})=0,
\end{gathered}
$$

where the displacement field $\mathbf{D}$ is related to the electric field $\mathbf{E}$ and the polarization field $\mathbf{P}$ according to

$$
\mathbf{D}(\mathbf{r})=\varepsilon_{0} \mathbf{E}(\mathbf{r})+\mathbf{P}(\mathbf{r}),
$$

and for nonmagnetic matter it may be assumed that

$$
\mathbf{H}(\mathbf{r})=\frac{1}{\mu_{0}} \mathbf{B}(\mathbf{r}) .
$$

Let us restrict our attention to isotropic but arbitrarily inhomogeneous media and assume that the polarization linearly and locally responds to the electric field. In this case, the most general relation between the polarization and the electric field which is in agreement with the causality principle and the dissipation-fluctuation theorem is

$$
\mathbf{P}(\mathbf{r}, t)=\varepsilon_{0} \int_{0}^{\infty} \mathrm{d} \tau \chi(\mathbf{r}, \tau) \mathbf{E}(\mathbf{r}, t-\tau)+\mathbf{P}_{\mathrm{n}}(\mathbf{r}, t),
$$

where $\chi(\mathbf{r}, \tau)$ is the dielectric susceptibility as a function of space and time, and $\mathbf{P}_{\mathrm{n}}$ is the (noise) polarization associated with absorption. 
Substitution of this expression into Eq. (5) together with Fourier transformation converts this equation to

$$
\underline{\mathbf{D}}(\mathbf{r}, \omega)=\varepsilon_{0} \varepsilon(\mathbf{r}, \omega) \underline{\mathbf{E}}(\mathbf{r}, \omega)+\underline{\mathbf{P}}_{\mathbf{n}}(\mathbf{r}, \omega),
$$

where

$$
\varepsilon(\mathbf{r}, \omega)=1+\int_{0}^{\infty} \mathrm{d} \tau \chi(\mathbf{r}, \tau) e^{i \omega \tau}
$$

is the (relative) permittivity, and the Maxwell equations (11)-(传) read in the Fourier domain as

$$
\begin{gathered}
\boldsymbol{\nabla} \cdot \underline{\mathbf{B}}(\mathbf{r}, \omega)=0, \\
\boldsymbol{\nabla} \times \underline{\mathbf{E}}(\mathbf{r}, \omega)=i \omega \underline{\mathbf{B}}(\mathbf{r}, \omega), \\
\varepsilon_{0} \boldsymbol{\nabla} \cdot \varepsilon(\mathbf{r}, \omega) \underline{\mathbf{E}}(\mathbf{r}, \omega)=\underline{\rho}_{\mathrm{n}}(\mathbf{r}, \omega), \\
\boldsymbol{\nabla} \times \underline{\mathbf{B}}(\mathbf{r}, \omega)+i \frac{\omega}{c^{2}} \varepsilon(\mathbf{r}, \omega) \underline{\mathbf{E}}(\mathbf{r}, \omega)=\mu_{0} \underline{\mathbf{j}}_{\mathrm{n}}(\mathbf{r}, \omega)
\end{gathered}
$$

$\left(c^{-2}=\varepsilon_{0} \mu_{0}\right)$. $\mathrm{f}$ Here we have introduced the noise charge density

$$
\underline{\rho}_{\mathrm{n}}(\mathbf{r}, \omega)=-\nabla \cdot \underline{\mathbf{P}}_{\mathrm{n}}(\mathbf{r}, \omega)
$$

and the noise current density

$$
\underline{\mathbf{j}}_{\mathrm{n}}(\mathbf{r}, \omega)=-i \omega \underline{\mathbf{P}}_{\mathrm{n}}(\mathbf{r}, \omega),
$$

which obey the continuity equation

$$
\nabla \cdot \underline{\mathbf{j}}_{\mathrm{n}}(\mathbf{r}, \omega)=i \omega \underline{\rho}_{\mathrm{n}}(\mathbf{r}, \omega) .
$$

According to Eq. (9), the permittivity $\epsilon(\mathbf{r}, \omega)$ is a complex function of frequency,

$$
\epsilon(\mathbf{r}, \omega)=\epsilon^{\prime}(\mathbf{r}, \omega)+i \epsilon^{\prime \prime}(\mathbf{r}, \omega) .
$$

The real and imaginary parts, which are responsible for dispersion and absorption respectively, are uniquely related to each other through the Kramers-Kronig relations

$$
\begin{aligned}
& \varepsilon^{\prime}(\mathbf{r}, \omega)-1=\frac{\mathcal{P}}{\pi} \int \mathrm{d} \omega^{\prime} \frac{\varepsilon^{\prime \prime}\left(\mathbf{r}, \omega^{\prime}\right)}{\omega^{\prime}-\omega}, \\
& \varepsilon^{\prime \prime}(\mathbf{r}, \omega)=-\frac{\mathcal{P}}{\pi} \int \mathrm{d} \omega^{\prime} \frac{\varepsilon^{\prime}\left(\mathbf{r}, \omega^{\prime}\right)-1}{\omega^{\prime}-\omega}
\end{aligned}
$$

$(\mathcal{P}$, principal value). Further, $\varepsilon(\mathbf{r}, \omega)$ as a function of complex $\omega$ satisfies the relation

$$
\varepsilon\left(\mathbf{r},-\omega^{*}\right)=\varepsilon^{*}(\mathbf{r}, \omega)
$$

\footnotetext{
${ }^{1}$ Here and in the following the Fourier transform $\underline{F}(\omega)$ of a real function $F(t)$ is defined according to the relation $F(t)=\int_{0}^{\infty} \mathrm{d} \omega \underline{F}(\omega) e^{-i \omega t}+$ c.c..
} 
and is holomorphic in the upper complex half-plane without zeros. In particular, it approaches unity in the high-frequency limit, i.e, $\varepsilon(\mathbf{r}, \omega) \rightarrow 1$ if $|\omega| \rightarrow \infty$.

The Maxwell equations (11) and (13) imply that $\underline{\mathbf{E}}(\mathbf{r}, \omega)$ obeys the partial differential equation

$$
\nabla \times \nabla \times \underline{\mathbf{E}}(\mathbf{r}, \omega)-\frac{\omega^{2}}{c^{2}} \varepsilon(\mathbf{r}, \omega) \underline{\mathbf{E}}(\mathbf{r}, \omega)=i \omega \mu_{0} \underline{\mathbf{j}}_{\mathbf{n}}(\mathbf{r}, \omega),
$$

whose solution can be represented in the form

$$
\underline{\mathbf{E}}(\mathbf{r}, \omega)=i \mu_{0} \omega \int \mathrm{d}^{3} \mathbf{r}^{\prime} \boldsymbol{G}\left(\mathbf{r}, \mathbf{r}^{\prime}, \omega\right) \cdot \underline{\mathbf{j}}_{\mathrm{n}}\left(\mathbf{r}^{\prime}, \omega\right),
$$

where the Green tensor $\boldsymbol{G}\left(\mathbf{r}, \mathbf{r}^{\prime}, \omega\right)$ has to be determined from the equation

$$
\boldsymbol{\nabla} \times \boldsymbol{\nabla} \times \boldsymbol{G}\left(\mathbf{r}, \mathbf{r}^{\prime}, \omega\right)-\frac{\omega^{2}}{c^{2}} \varepsilon(\mathbf{r}, \omega) \boldsymbol{G}\left(\mathbf{r}, \mathbf{r}^{\prime}, \omega\right)=\boldsymbol{\delta}\left(\mathbf{r}-\mathbf{r}^{\prime}\right)
$$

together with the boundary condition at infinity. In Cartesian coordinates, Eq.(23) reads

$$
\left[\left(\partial_{i}^{r} \partial_{m}^{r}-\delta_{i m} \Delta^{r}\right)-\delta_{i m} \frac{\omega^{2}}{c^{2}} \varepsilon(\mathbf{r}, \omega)\right] G_{m j}\left(\mathbf{r}, \mathbf{r}^{\prime}, \omega\right)=\delta_{i j} \delta\left(\mathbf{r}-\mathbf{r}^{\prime}\right)
$$

$\left(\partial_{i}^{r}=\partial / \partial x_{i}\right)$, where over repeated vector-component indices is summed. The Green tensor has the properties that

$$
\begin{gathered}
G_{i j}\left(\mathbf{r}, \mathbf{r}^{\prime}, \omega\right)^{*}=G_{i j}\left(\mathbf{r}, \mathbf{r}^{\prime},-\omega\right), \\
G_{j i}\left(\mathbf{r}^{\prime}, \mathbf{r}, \omega\right)=G_{i j}\left(\mathbf{r}, \mathbf{r}^{\prime}, \omega\right),
\end{gathered}
$$

and

$$
\int \mathrm{d}^{3} \mathbf{s} \frac{\omega^{2}}{c^{2}} \varepsilon^{\prime \prime}(\mathbf{s}, \omega) G_{i k}(\mathbf{r}, \mathbf{s}, \omega) G_{j k}^{*}\left(\mathbf{r}^{\prime}, \mathbf{s}, \omega\right)=\operatorname{Im} G_{i j}\left(\mathbf{r}, \mathbf{r}^{\prime}, \omega\right) .
$$

The property (25) is a direct consequence of the corresponding relation (20) for the permittivity, Eq. (26) is the so-called reciprocity relation, and (27) is proved in [27.

The Fourier components of the magnetic induction, $\underline{\mathbf{B}}(\mathbf{r}, \omega)$, and the displacement field, $\underline{\mathbf{D}}(\mathbf{r}, \omega)$, are directly related to the Fourier components of the electric field, $\underline{\mathbf{E}}(\mathbf{r}, \omega)$,

$$
\begin{gathered}
\underline{\mathbf{B}}(\mathbf{r}, \omega)=(i \omega)^{-1} \nabla \times \underline{\mathbf{E}}(\mathbf{r}, \omega), \\
\underline{\mathbf{D}}(\mathbf{r}, \omega)=\left(\mu_{0} \omega^{2}\right)^{-1} \nabla \times \boldsymbol{\nabla} \times \underline{\mathbf{E}}(\mathbf{r}, \omega)
\end{gathered}
$$

[see Eqs. (11), (8), (15), and (21)], and $\underline{\mathbf{E}}(\mathbf{r}, \omega)$ is determined, according to Eq. (22), by $\mathbf{j}_{\mathrm{n}}(\mathbf{r}, \omega)$. The continuous set of (complex) fields $\mathbf{j}_{\mathrm{n}}(\mathbf{r}, \omega)$ [or, equivalently $\mathbf{P}_{\mathrm{n}}(\mathbf{r}, \omega)$ ] can therefore be regarded as playing the role of the set of dynamical variables of the system composed of the electromagnetic field and the medium (including the dissipative system). For the following it is convenient to split off some factor from $\mathbf{P}_{\mathrm{n}}(\mathbf{r}, \omega)$ and to define the fundamental dynamical variables $\mathbf{f}(\mathbf{r}, \omega)$ as follows:

$$
\underline{\mathbf{P}}_{\mathrm{n}}(\mathbf{r}, \omega)=i \sqrt{\frac{\hbar \varepsilon_{0}}{\pi} \varepsilon^{\prime \prime}(\mathbf{r}, \omega)} \mathbf{f}(\mathbf{r}, \omega) \text {. }
$$




\section{$2.2 \quad$ Field quantization}

The transition from classical to quantum theory now consists in the replacement of the classical fields $\mathbf{f}(\mathbf{r}, \omega)$ and $\mathbf{f}^{*}(\mathbf{r}, \omega)$ by the operator-valued bosonic fields $\hat{\mathbf{f}}(\mathbf{r}, \omega)$ and $\hat{\mathbf{f}}^{\dagger}(\mathbf{r}, \omega)$ respectively, which are associated with the elementary excitations of the composed system in linear approximation. Thus the commutation relations are

$$
\begin{gathered}
{\left[\hat{f}_{k}(\mathbf{r}, \omega), \hat{f}_{k^{\prime}}^{\dagger}\left(\mathbf{r}^{\prime}, \omega^{\prime}\right)\right]=\delta_{k k^{\prime}} \delta\left(\mathbf{r}-\mathbf{r}^{\prime}\right) \delta\left(\omega-\omega^{\prime}\right),} \\
{\left[\hat{f}_{k}(\mathbf{r}, \omega), \hat{f}_{k^{\prime}}\left(\mathbf{r}^{\prime}, \omega^{\prime}\right)\right]=0,}
\end{gathered}
$$

and the Hamiltonian of the composed system is

$$
\hat{H}=\int \mathrm{d}^{3} \mathbf{r} \int_{0}^{\infty} \mathrm{d} \omega \hbar \omega \hat{\mathbf{f}}^{\dagger}(\mathbf{r}, \omega) \cdot \hat{\mathbf{f}}(\mathbf{r}, \omega)
$$

Recalling Eqs. (15) and (30) and replacing $\mathbf{E}(\mathbf{r}, \omega)$ [Eq. (22)], B(r,w) [Eq. (28)], and $\mathbf{D}(\mathbf{r}, \omega)[\mathrm{Eq} .(29)]$ by the quantum-mechanical operators, we find that

$$
\begin{gathered}
\underline{\hat{\mathbf{E}}}(\mathbf{r}, \omega)=i \sqrt{\frac{\hbar}{\pi \varepsilon_{0}}} \frac{\omega^{2}}{c^{2}} \int \mathrm{d}^{3} \mathbf{r}^{\prime} \sqrt{\varepsilon^{\prime \prime}\left(\mathbf{r}^{\prime}, \omega\right)} \boldsymbol{G}\left(\mathbf{r}, \mathbf{r}^{\prime}, \omega\right) \cdot \hat{\mathbf{f}}\left(\mathbf{r}^{\prime}, \omega\right), \\
\underline{\hat{\mathbf{B}}}(\mathbf{r}, \omega)=(i \omega)^{-1} \nabla \times \underline{\hat{\mathbf{E}}}(\mathbf{r}, \omega),
\end{gathered}
$$

and

$$
\underline{\hat{\mathbf{D}}}(\mathbf{r}, \omega)=\left(\mu_{0} \omega^{2}\right)^{-1} \nabla \times \nabla \times \underline{\hat{\mathbf{E}}}(\mathbf{r}, \omega),
$$

from which the electromagnetic field operators in the Schrödinger picture are obtained by integration over $\omega$ :

$$
\begin{gathered}
\hat{\mathbf{E}}(\mathbf{r}) \equiv \hat{\mathbf{E}}_{\mathrm{m}}(\mathbf{r})=\int_{0}^{\infty} \mathrm{d} \omega \underline{\hat{\mathbf{E}}}(\mathbf{r}, \omega)+\text { H.c. } \\
\hat{\mathbf{B}}(\mathbf{r})=\int_{0}^{\infty} \mathrm{d} \omega \underline{\hat{\mathbf{B}}}(\mathbf{r}, \omega)+\text { H.c. }
\end{gathered}
$$

and $\left.\right|^{2}$

$$
\hat{\mathbf{D}}(\mathbf{r}) \equiv \hat{\mathbf{D}}_{\mathrm{m}}(\mathbf{r})=\hat{\mathbf{D}}_{\mathrm{m}}^{\perp}(\mathbf{r})=\int_{0}^{\infty} \mathrm{d} \omega \underline{\underline{\mathbf{D}}}(\mathbf{r}, \omega)+\text { H.c. }
$$

In this way, the electromagnetic field is expressed in terms of the classical Green tensor $\boldsymbol{G}\left(\mathbf{r}, \mathbf{r}^{\prime}, \omega\right)$ satisfying the generalized Helmholtz equation (23) and the continuum of

${ }^{2}$ The longitudinal $\left(\mathbf{F}^{\|}\right)$and transverse $\left(\mathbf{F}^{\perp}\right)$ parts of a vector field $\mathbf{F}$ are defined by $\mathbf{F}^{\|(\perp)}(\mathbf{r})=$ $\int \mathbf{d}^{3} \mathbf{r}^{\prime} \boldsymbol{\delta}^{\|(\perp)}\left(\mathbf{r}-\mathbf{r}^{\prime}\right) \mathbf{F}\left(\mathbf{r}^{\prime}\right)$, with $\boldsymbol{\delta}^{(\|)}(\mathbf{r})$ and $\boldsymbol{\delta}^{(\perp)}(\mathbf{r})$ being the longitudinal and transverse tensor-valued $\delta$-functions respectively. 
the fundamental bosonic field variables $\hat{\mathbf{f}}(\mathbf{r}, \omega)$ [and $\hat{\mathbf{f}}^{\dagger}(\mathbf{r}, \omega)$ ]. All the information about the dielectric matter (such as its formation in space and its dispersive and absorptive properties) is contained [via the permittivity $\varepsilon(\mathbf{r}, \omega)$ ] in the Green tensor of the classical problem. Eqs. (37) - (39), together with Eqs. (34) - (36) can be considered as the generalization of the familiar mode decomposition.

The quantization scheme meets the basic requirements of quantum electrodynamics. So it can be shown by using very general properties of the permittivity and the Green tensor that $\hat{\mathbf{E}}$ and $\hat{\mathbf{B}}$ satisfy the correct (equal-time) commutation relations 27

$$
\begin{gathered}
{\left[\hat{E}_{k}(\mathbf{r}), \hat{E}_{k^{\prime}}\left(\mathbf{r}^{\prime}\right)\right]=0=\left[\hat{B}_{k}(\mathbf{r}), \hat{B}_{k^{\prime}}\left(\mathbf{r}^{\prime}\right)\right],} \\
{\left[\varepsilon_{0} \hat{E}_{k}(\mathbf{r}), \hat{B}_{k^{\prime}}\left(\mathbf{r}^{\prime}\right)\right]=-i \hbar \epsilon_{k k^{\prime} l} \partial_{l}^{r} \delta\left(\mathbf{r}-\mathbf{r}^{\prime}\right) .}
\end{gathered}
$$

Obviously, the electromagnetic field operators in the Heisenberg picture satisfy the

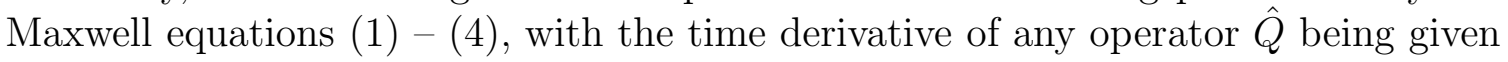
by

$$
\dot{\hat{Q}}=(i \hbar)^{-1}[\hat{Q}, \hat{H}]
$$

where $\hat{H}$ is the Hamiltonian (33),

Further, scalar $(\hat{\varphi})$ and vector $(\hat{\mathbf{A}})$ potentials can be introduced and expressed in terms of the fundamental bosonic fields. In particular, the potentials in the Coulomb gauge are defined by

$$
\begin{gathered}
-\nabla \hat{\varphi}(\mathbf{r})=\hat{\mathbf{E}}^{\|}(\mathbf{r}), \\
\hat{\mathbf{A}}(\mathbf{r})=\int_{0}^{\infty} \mathrm{d} \omega \underline{\hat{\mathbf{A}}}(\mathbf{r}, \omega)+\text { H.c. },
\end{gathered}
$$

where

$$
\underline{\hat{\mathbf{A}}}(\mathbf{r}, \omega)=(i \omega)^{-1} \underline{\hat{\mathbf{E}}}^{\perp}(\mathbf{r}, \omega)
$$

The canonically conjugated momentum field with respect to $\hat{\mathbf{A}}(\mathbf{r})$ is

$$
\hat{\boldsymbol{\Pi}}(\mathbf{r})=-i \varepsilon_{0} \int_{0}^{\infty} \mathrm{d} \omega \omega \underline{\hat{\mathbf{A}}}(\mathbf{r}, \omega)+\text { H.c. },
$$

and it is not difficult to verify that $\hat{\mathbf{\Pi}}=-\varepsilon_{0} \hat{\mathbf{E}}^{\perp}, \nabla \times \hat{\mathbf{A}}=\hat{\mathbf{B}}$, and $-\dot{\hat{\mathbf{A}}}-\boldsymbol{\nabla} \hat{\varphi}(\mathbf{r})=\hat{\mathbf{E}}$. In addition, $\hat{\mathbf{A}}$ and $\hat{\boldsymbol{\Pi}}$ satisfy the well-known commutation relations

$$
\begin{gathered}
{\left[\hat{A}_{k}(\mathbf{r}), \hat{A}_{k^{\prime}}\left(\mathbf{r}^{\prime}\right)\right]=0=\left[\hat{\Pi}_{k}(\mathbf{r}), \hat{\Pi}_{k^{\prime}}\left(\mathbf{r}^{\prime}\right)\right],} \\
{\left[\hat{A}_{k}(\mathbf{r}), \hat{\Pi}_{k^{\prime}}\left(\mathbf{r}^{\prime}\right)\right]=i \hbar \delta_{k k^{\prime}}^{\perp}\left(\mathbf{r}-\mathbf{r}^{\prime}\right) .}
\end{gathered}
$$




\section{Interaction of the medium-assisted quantized electromagnetic field with charged particles}

The interaction of the quantized electromagnetic field with atoms placed inside a dielectric medium or near dielectric bodies can be strongly influenced by the dielectric medium. A well-known example is the dependence of the spontaneous decay rate of an excited atom on the properties of an dielectric environment. In order to study such and related phenomena, the Hamiltonian (33) must be supplemented with the the Hamiltonian of additional charged particles and their interaction energy with the medium-assisted electromagnetic field.

\subsection{The minimal-coupling Hamiltonian}

Applying the minimal-coupling scheme, we may write the the total Hamiltonian in the form

$$
\begin{gathered}
\hat{H}=\int \mathrm{d}^{3} \mathbf{r} \int_{0}^{\infty} \mathrm{d} \omega \hbar \omega \hat{\mathbf{f}}^{\dagger}(\mathbf{r}, \omega) \cdot \hat{\mathbf{f}}(\mathbf{r}, \omega)+\sum_{\alpha} \frac{1}{2 m_{\alpha}}\left[\hat{\mathbf{p}}_{\alpha}-q_{\alpha} \hat{\mathbf{A}}\left(\hat{\mathbf{r}}_{\alpha}\right)\right]^{2} \\
+\frac{1}{2} \int \mathrm{d}^{3} \mathbf{r} \hat{\rho}_{\mathrm{A}}(\mathbf{r}) \hat{\varphi}_{\mathrm{A}}(\mathbf{r})+\int \mathrm{d}^{3} \mathbf{r} \hat{\rho}_{\mathrm{A}}(\mathbf{r}) \hat{\varphi}(\mathbf{r}),
\end{gathered}
$$

where $\hat{\mathbf{r}}_{\alpha}$ is the position operator and $\hat{\mathbf{p}}_{\alpha}$ is the canonical momentum operator of the $\alpha$ th (non-relativistic) particle of charge $q_{\alpha}$ and mass $m_{\alpha}$. The Hamiltonian (49) consists of four terms. The first term is the energy of the electromagnetic field and the medium (including the dissipative system), as introduced in Eq. (33). The second term is the kinetic energy of the charged particles, and the third term is their Coulomb energy, where the corresponding scalar potential $\hat{\varphi}_{\mathrm{A}}$ is given by

$$
\hat{\varphi}_{\mathrm{A}}(\mathbf{r})=\int \mathrm{d}^{3} \mathbf{r}^{\prime} \frac{\hat{\rho}_{\mathrm{A}}\left(\mathbf{r}^{\prime}\right)}{4 \pi \epsilon_{0}\left|\mathbf{r}-\hat{\mathbf{r}}^{\prime}\right|},
$$

with

$$
\hat{\rho}_{\mathrm{A}}(\mathbf{r})=\sum_{\alpha} q_{\alpha} \delta\left(\mathbf{r}-\hat{\mathbf{r}}_{\alpha}\right)
$$

being the charge density. The last term is the Coulomb energy of interaction of the particles with the medium. From Eq. (49) it follows that the interaction Hamiltonian reads

$$
\hat{H}_{\mathrm{int}}=-\sum_{\alpha} \frac{1}{m_{\alpha}}\left[\hat{\mathbf{p}}_{\alpha}-\frac{1}{2} q_{\alpha} \hat{\mathbf{A}}\left(\hat{\mathbf{r}}_{\alpha}\right)\right] q_{\alpha} \hat{\mathbf{A}}\left(\hat{\mathbf{r}}_{\alpha}\right)+\int \mathrm{d}^{3} \mathbf{r} \hat{\rho}_{\mathrm{A}}(\mathbf{r}) \hat{\varphi}(\mathbf{r}) .
$$

Note that in Eq. (52) the scalar potential $\hat{\varphi}$ and the vector potential $\hat{\mathbf{A}}$ must be thought of as being expressed, on using Eqs. (43), (44), (45) together with Eqs. (34) and (37), in terms of the fundamental fields $\hat{\mathbf{f}}(\mathbf{r}, \omega)$ and $\hat{\mathbf{f}}^{\dagger}(\mathbf{r}, \omega)$. 
In a straightforward but somewhat lengthy calculation it can be shown that both the operator-valued Maxwell equations

$$
\begin{gathered}
\boldsymbol{\nabla} \cdot \hat{\mathbf{B}}(\mathbf{r})=0, \\
\nabla \times \hat{\mathbf{E}}(\mathbf{r})+\dot{\hat{\mathbf{B}}}(\mathbf{r})=0, \\
\nabla \cdot \hat{\mathbf{D}}(\mathbf{r})=\hat{\rho}_{\mathrm{A}}(\mathbf{r}), \\
\nabla \times \hat{\mathbf{H}}(\mathbf{r})-\dot{\hat{\mathbf{D}}}(\mathbf{r})=\hat{\mathbf{j}}_{\mathrm{A}}(\mathbf{r}),
\end{gathered}
$$

and the operator-valued Newtonian equation of motion

$$
\begin{gathered}
\dot{\hat{\mathbf{r}}}_{\alpha}=\frac{1}{m_{\alpha}}\left[\hat{\mathbf{p}}_{\alpha}-q_{\alpha} \hat{\mathbf{A}}\left(\hat{\mathbf{r}}_{\alpha}\right)\right], \\
m_{\alpha} \ddot{\hat{\mathbf{r}}}_{\alpha}=q_{\alpha}\left[\hat{\mathbf{E}}\left(\hat{\mathbf{r}}_{\alpha}\right)+\frac{1}{2}\left(\dot{\hat{\mathbf{r}}}_{\alpha} \times \hat{\mathbf{B}}\left(\hat{\mathbf{r}}_{\alpha}\right)-\hat{\mathbf{B}}\left(\hat{\mathbf{r}}_{\alpha}\right) \times \dot{\hat{\mathbf{r}}}_{\alpha}\right)\right] .
\end{gathered}
$$

are fulfilled. In Eq. (56), the atomic current density $\hat{\mathbf{j}}_{\mathrm{A}}(\mathbf{r})$ reads

$$
\hat{\mathbf{j}}_{\mathrm{A}}(\mathbf{r})=\frac{1}{2} \sum_{\alpha} q_{\alpha}\left[\delta\left(\mathbf{r}-\hat{\mathbf{r}}_{\alpha}\right), \dot{\hat{\mathbf{r}}}_{\alpha}\right]_{+},
$$

where $[,]_{+}$denotes the anticommutator. Note that compared with Eqs. (37) and (39), the electric and displacement fields now contain additional longitudinal parts that result from the charge distribution $\hat{\rho}_{\mathrm{A}}(\mathbf{r})$, i.e.,

$$
\begin{gathered}
\hat{\mathbf{E}}(\mathbf{r})=\hat{\mathbf{E}}_{\mathrm{m}}(\mathbf{r})-\boldsymbol{\nabla} \hat{\varphi}_{\mathrm{A}}(\mathbf{r})=\left[\int_{0}^{\infty} \mathrm{d} \omega \underline{\hat{\mathbf{E}}}(\mathbf{r}, \omega)+\text { H.c. }\right]-\nabla \hat{\varphi}_{\mathrm{A}}(\mathbf{r}), \\
\hat{\mathbf{D}}(\mathbf{r})=\hat{\mathbf{D}}_{\mathrm{m}}(\mathbf{r})-\varepsilon_{0} \boldsymbol{\nabla} \hat{\varphi}_{\mathrm{A}}(\mathbf{r})=\left[\int_{0}^{\infty} \mathrm{d} \omega \underline{\hat{\mathbf{D}}}(\mathbf{r}, \omega)+\text { H.c. }\right]-\varepsilon_{0} \boldsymbol{\nabla} \hat{\varphi}_{\mathrm{A}}(\mathbf{r}) .
\end{gathered}
$$

The Maxwell equations (53) and (55) simply result from the definition of the field operators $\hat{\mathbf{B}}(\mathbf{r})$ [Eqs. (35) and (38)] and $\hat{\mathbf{D}}(\mathbf{r})$ [Eqs. (36), (50), and (61)] respectively. The other Maxwell equations (54) and (56) and the Newtonian equation of motion (57) and (58) follow from the Heisenberg equation of motion, Eq. (2.37), with the Hamiltonian $\hat{H}$ from Eq. (49).

\subsection{The multipolar-coupling Hamiltonian}

In the minimal-coupling scheme, the interaction Hamiltonian (52) is expressed in terms of the potentials of the medium-assisted electromagnetic field. With regard to (localized) atomic systems (atoms, molecules etc.) the interaction is commonly desired to be treated in terms of the electromagnetic field strengths and the atomic 
polarization and magnetization. This can be achieved by means of a unitary transformation.

Let us consider an atomic system localized at position $\mathbf{r}_{\mathrm{A}}$ and introduce the atomic polarization

$$
\hat{\mathbf{P}}_{\mathrm{A}}(\mathbf{r})=\sum_{\alpha} q_{\alpha}\left(\hat{\mathbf{r}}_{\alpha}-\mathbf{r}_{\mathrm{A}}\right) \int_{0}^{1} \mathrm{~d} \lambda \delta\left[\mathbf{r}-\mathbf{r}_{\mathrm{A}}-\lambda\left(\hat{\mathbf{r}}_{\alpha}-\mathbf{r}_{\mathrm{A}}\right)\right],
$$

so that the charge density (51) can be rewritten as

$$
\hat{\rho}_{\mathrm{A}}(\mathbf{r})=\sum_{\alpha} q_{\alpha} \delta\left(\mathbf{r}-\hat{\mathbf{r}}_{\mathrm{A}}\right)-\boldsymbol{\nabla} \cdot \hat{\mathbf{P}}_{\mathrm{A}}(\mathbf{r}) .
$$

In order to perform the transition from the minimal-coupling scheme to the multipolar-coupling scheme, we apply to the variables the unitary operator

$$
\hat{U}=\exp \left[\frac{i}{\hbar} \int \mathrm{d}^{3} \mathbf{r} \hat{\mathbf{P}}_{\mathrm{A}}(\mathbf{r}) \cdot \hat{\mathbf{A}}(\hat{\mathbf{r}})\right] .
$$

It is not difficult to prove that the following transformation rules are valid:

$$
\begin{gathered}
\hat{\mathbf{r}}_{\alpha}^{\prime}=\hat{U} \hat{\mathbf{r}}_{\alpha} \hat{U}^{\dagger}=\hat{\mathbf{r}}_{\alpha}, \\
\hat{\mathbf{p}}_{\alpha}^{\prime}=\hat{U} \hat{\mathbf{p}}_{\alpha} \hat{U}^{\dagger}=\hat{\mathbf{p}}_{\alpha}-q_{\alpha} \hat{\mathbf{A}}\left(\hat{\mathbf{r}}_{\alpha}\right)-\int \mathrm{d}^{3} \mathbf{r} \hat{\mathbf{n}}_{\alpha}(\mathbf{r}) \times \hat{\mathbf{B}}(\mathbf{r}), \\
\hat{\mathbf{f}}^{\prime}(\mathbf{r}, \omega)=\hat{U} \hat{\mathbf{f}}(\mathbf{r}, \omega) \hat{U}^{\dagger} \\
=\hat{\mathbf{f}}(\mathbf{r}, \omega)-\frac{i}{\hbar} \sqrt{\frac{\hbar}{\pi \varepsilon_{0}} \varepsilon^{\prime \prime}(\mathbf{r}, \omega)} \frac{\omega}{c^{2}} \int \mathrm{d}^{3} \mathbf{r}^{\prime} \hat{\mathbf{P}}_{\mathrm{A}}^{\perp}\left(\mathbf{r}^{\prime}\right) \cdot G^{*}\left(\mathbf{r}^{\prime}, \mathbf{r}, \omega\right),
\end{gathered}
$$

where the abbreviation

$$
\hat{\mathbf{n}}_{\alpha}(\mathbf{r})=q_{\alpha}\left(\hat{\mathbf{r}}_{\alpha}-\mathbf{r}_{\mathrm{A}}\right) \int_{0}^{1} \mathrm{~d} \lambda \lambda \delta\left[\mathbf{r}-\mathbf{r}_{\mathrm{A}}-\lambda\left(\hat{\mathbf{r}}_{\alpha}-\mathbf{r}_{\mathrm{A}}\right)\right]
$$

has been used. Employing equations (65) - (67), we can express the Hamiltonian $\hat{H}$ in Eq. (49) as the unitary transform of a new Hamiltonian $\hat{\mathcal{H}}$,

$$
\hat{H}=\hat{U} \hat{\mathcal{H}} \hat{U}^{\dagger},
$$

where

$$
\begin{aligned}
\hat{\mathcal{H}}=\int \mathrm{d}^{3} \mathbf{r} \int_{0}^{\infty} \mathrm{d} \omega \hbar \omega \hat{\mathbf{f}}^{\dagger}(\mathbf{r}, \omega) \cdot \hat{\mathbf{f}}(\mathbf{r}, \omega)+\sum_{\alpha} \frac{1}{2 m_{\alpha}}\left[\hat{\mathbf{p}}_{\alpha}+\int \mathrm{d}^{3} \mathbf{r} \hat{\mathbf{n}}_{\alpha}(\mathbf{r}) \times \hat{\mathbf{B}}(\mathbf{r})\right]^{2} \\
+\frac{1}{2} \int \mathrm{d}^{3} \mathbf{r} \hat{\rho}_{\mathrm{A}}(\mathbf{r}) \hat{\varphi}_{\mathrm{A}}(\mathbf{r})+\frac{1}{2 \varepsilon_{0}} \int \mathrm{d}^{3} \mathbf{r} \hat{\mathbf{P}}_{\mathrm{A}}^{\perp}(\mathbf{r}) \cdot \hat{\mathbf{P}}_{\mathrm{A}}^{\perp}(\mathbf{r}) \\
\quad-\int \mathrm{d}^{3} \mathbf{r} \hat{\mathbf{P}}_{\mathrm{A}}^{\perp}(\mathbf{r}) \cdot \hat{\mathbf{E}}_{\mathrm{m}}(\mathbf{r})+\int \mathrm{d}^{3} \mathbf{r} \hat{\rho}_{\mathrm{A}}(\mathbf{r}) \hat{\varphi}(\mathbf{r})
\end{aligned}
$$


In particular when the charged particles form a neutral atomic system $\left(\sum_{\alpha} q_{\alpha}=0\right)$, then Eq. (70) takes the form of

$$
\begin{gathered}
\hat{\mathcal{H}}=\int \mathrm{d}^{3} \mathbf{r} \int_{0}^{\infty} \mathrm{d} \omega \hbar \omega \hat{\mathbf{f}}^{\dagger}(\mathbf{r}, \omega) \cdot \hat{\mathbf{f}}(\mathbf{r}, \omega)+\sum_{\alpha} \frac{1}{2 m_{\alpha}}\left[\hat{\mathbf{p}}_{\alpha}+\int \mathrm{d}^{3} \mathbf{r} \hat{\mathbf{n}}_{\alpha}(\mathbf{r}) \times \hat{\mathbf{B}}(\mathbf{r})\right]^{2} \\
+\frac{1}{2 \varepsilon_{0}} \int \mathrm{d}^{3} \mathbf{r} \hat{\mathbf{P}}_{\mathrm{A}}(\mathbf{r}) \cdot \hat{\mathbf{P}}_{\mathrm{A}}(\mathbf{r})-\int \mathrm{d}^{3} \mathbf{r} \hat{\mathbf{P}}_{\mathrm{A}}(\mathbf{r}) \cdot \hat{\mathbf{E}}_{\mathrm{m}}(\mathbf{r}) .
\end{gathered}
$$

Note that the last term on the right-hand side in Eq. (71) describes the interaction between the atomic polarization and the whole medium-assisted electric field. Introducing the polarization of the medium

$$
\hat{\mathbf{P}}_{\mathrm{m}}=\hat{\mathbf{D}}-\varepsilon_{0} \hat{\mathbf{E}}=\hat{\mathbf{D}}_{\mathrm{m}}-\varepsilon_{0} \hat{\mathbf{E}}_{\mathrm{m}}=\hat{\mathbf{D}}_{\mathrm{m}}^{\perp}-\varepsilon_{0} \hat{\mathbf{E}}_{\mathrm{m}}
$$

[see Eqs. (60) and (61] and recalling that $\hat{\mathbf{D}}_{\mathrm{m}}^{\perp}=\hat{\mathbf{D}}^{\perp}$, we may rewrite the last term in the transformed Hamiltonian (1) to obtain

$$
-\int d^{3} \mathbf{r} \hat{\mathbf{P}}_{\mathrm{A}}(\mathbf{r}) \cdot \hat{\mathbf{E}}_{\mathrm{m}}(\mathbf{r})=-\frac{1}{\varepsilon_{0}} \int \mathrm{d}^{3} \mathbf{r} \hat{\mathbf{P}}_{\mathrm{A}}(\mathbf{r}) \cdot \hat{\mathbf{D}}^{\perp}(\mathbf{r})+\frac{1}{\varepsilon_{0}} \int \mathrm{d}^{3} \mathbf{r} \hat{\mathbf{P}}_{\mathrm{A}}(\mathbf{r}) \cdot \hat{\mathbf{P}}_{\mathrm{m}}(\mathbf{r}) .
$$

Combining Eqs. (69), (71), and (73), we express in the original Hamiltonian the old variables in terms of the new ones. The result is the multipolar-coupling Hamiltonian

$$
\begin{aligned}
\hat{H}= & \int \mathrm{d}^{3} \mathbf{r} \int_{0}^{\infty} \mathrm{d} \omega \hbar \omega \hat{\mathbf{f}}^{\prime \dagger}(\mathbf{r}, \omega) \cdot \hat{\mathbf{f}}^{\prime}(\mathbf{r}, \omega)+\sum_{\alpha} \frac{1}{2 m_{\alpha}}\left[\hat{\mathbf{p}}_{\alpha}^{\prime}+\int \mathrm{d}^{3} \mathbf{r} \hat{\mathbf{n}}_{\alpha}(\mathbf{r}) \times \hat{\mathbf{B}}(\mathbf{r})\right]^{2} \\
& +\frac{1}{2 \varepsilon_{0}} \int \mathrm{d}^{3} \mathbf{r} \hat{\mathbf{P}}_{\mathrm{A}}(\mathbf{r}) \cdot \hat{\mathbf{P}}_{\mathrm{A}}(\mathbf{r})-\frac{1}{\varepsilon_{0}} \int \mathrm{d}^{3} \mathbf{r} \hat{\mathbf{P}}_{\mathrm{A}}(\mathbf{r}) \cdot \hat{\mathbf{D}}^{\prime \perp}(\mathbf{r})+\frac{1}{\varepsilon_{0}} \int \mathrm{d}^{3} \mathbf{r} \hat{\mathbf{P}}_{\mathrm{A}}(\mathbf{r}) \cdot \hat{\mathbf{P}}_{\mathrm{m}}(\mathbf{r}),
\end{aligned}
$$

where

$$
\hat{\mathbf{D}}^{\prime \perp}(\mathbf{r})=\hat{\mathbf{D}}^{\perp}(\mathbf{r})+\hat{\mathbf{P}}_{\mathrm{A}}^{\perp}(\mathbf{r}) .
$$

From Eq. (74) the interaction Hamiltonian is seen to be

$$
\begin{aligned}
\hat{H}_{\mathrm{int}}{ }^{\prime} & =-\frac{1}{\varepsilon_{0}} \int \mathrm{d}^{3} \mathbf{r} \hat{\mathbf{P}}_{\mathrm{A}}(\mathbf{r}) \cdot \hat{\mathbf{D}}^{\prime \perp}(\mathbf{r})+\frac{1}{\varepsilon_{0}} \int \mathrm{d}^{3} \mathbf{r} \hat{\mathbf{P}}_{\mathrm{A}}(\mathbf{r}) \cdot \hat{\mathbf{P}}_{\mathrm{m}}(\mathbf{r}) \\
& +\sum_{\alpha} \frac{1}{2 m_{\alpha}}\left[\hat{\mathbf{p}}_{\alpha}^{\prime}, \int \mathrm{d}^{3} \mathbf{r} \hat{\mathbf{n}}_{\alpha}(\mathbf{r}) \times \hat{\mathbf{B}}(\mathbf{r})\right]_{+}+\sum_{\alpha} \frac{1}{2 m_{\alpha}}\left[\int \mathrm{d}^{3} \mathbf{r} \hat{\mathbf{n}}_{\alpha}(\mathbf{r}) \times \hat{\mathbf{B}}(\mathbf{r})\right]^{2}
\end{aligned}
$$

The first term on the right-hand side in Eq. (76) describes the interaction of the polarization of the atomic system with the transverse part of the overall displacement field [cf. Eq. (75)]. The second term is a contact term between the medium polarization and the polarization of the atomic system. The last two terms refer to magnetic interactions. 


\section{Summary and outlook}

We have developed a general theory of the interaction of the quantized electromagnetic field with atoms in the presence of dispersing and absorbing dielectric bodies of given Kramers-Kronig consistent permittivities. The concept is based on a sourcequantity representation of the electromagnetic field, in which the electromagneticfield operators are expressed in terms of a continuous set of fundamental bosonic fields via the Green tensor of the classical problem. The theory, which is a natural extension of the standard concept of mode decomposition, gives a unified approach to the atom-field interaction, without any restriction to a particular frequency range.

The formalism has been applied quite recently to the problem of the spontaneous decay of an excited atom in the presence of dielectric bodies [28]. In particular, it has been shown that the temporal evolution of the atomic upper-state-probability amplitude $C_{u}(t)$ obeys the integral equation

$$
C_{u}(t)=1+\int_{0}^{t} \mathrm{~d} t^{\prime} \bar{K}\left(t-t^{\prime}\right) C_{u}\left(t^{\prime}\right)
$$

where the kernel function is determined by the Green tensor (at the atomic position $\mathbf{r}_{\mathrm{A}}$ ) as follows:

$$
K\left(t-t^{\prime}\right)=\frac{\omega_{\mathrm{A}}^{2} \mu_{i} \mu_{j}}{\hbar \pi \varepsilon_{0} c^{2}} \int_{0}^{\infty} \mathrm{d} \omega \frac{\operatorname{Im} G_{i j}\left(\mathbf{r}_{\mathrm{A}}, \mathbf{r}_{\mathrm{A}}, \omega\right)}{i\left(\omega-\omega_{\mathrm{A}}\right)}\left[e^{-i\left(\omega-\omega_{\mathrm{A}}\right)\left(t-t^{\prime}\right)}-1\right]
$$

( $\omega_{\mathrm{A}}$, transition frequency; $\mu_{i}$, transition dipole moment). It is worth noting that the integral equation (77) applies to the spontaneous decay of an atom in the presence of an arbitrary configuration of dispersing and absorbing dielectric bodies. All the matter parameters that are relevant for the atomic evolution are contained, via the Green tensor, in the kernel function (78). It should be pointed out that the Green tensor has been available for a large variety of configurations such as planarly, spherically, and cylindrically multilayered media [29]. A first evaluation of the integral equation (77) for the case of the atom being placed at the centre of a spherical micro-cavity whose wall is modeled by a band-gap dielectric of Lorentz type has been given in reference 28] to which the reader is referred for details.

\section{References}

[1] J.M. Jauch and K.M. Watson, Phys. Rev. 74, 950 (1948); ibid. 74, 1485 (1948).

[2] Y.R. Shen, Phys. Rev. 155, 921 (1967).

[3] C.K. Carniglia and L. Mandel, Phys. Rev. D 3, 280 (1971). 
[4] I. Bialynicki-Birula and J.B. Brojan, Phys. Rev. D 5, 485 (1972).

[5] I. Abram, Phys. Rev. A 35, 4661 (1987).

[6] Z. Bialynicki-Birula and I. Bialynicki-Birula, J. Opt. Soc. Am. B 4, 1621 (1987).

[7] L. Knöll, W. Vogel, and D.-G. Welsch, Phys. Rev. A 36, 3803 (1987).

[8] T.A.B. Kennedy and E.M. Wright, Phys. Rev. A 38, 212 (1988).

[9] H. Khosravi and R. Loudon, Proc. R. Soc. London Ser. A 433, 337 (1991); ibid. 436, 373 (1992).

[10] R.J. Glauber and M. Lewenstein, Phys. Rev. A 43, 467 (1991).

[11] B.J. Dalton, E.S. Guerra, and P.L. Knight, Phys. Rev. A 54, 2292 (1996).

[12] M. Bordag, K. Kirsten, and D.V. Vassilevich, J. Phys. A: Math. Gen. 31, 2381 (1998).

[13] B.J. Dalton, S.M. Barnett, and P.L. Knight, J. Mod. Opt. 46, 1315 (1999); ibid. 46, 1495 (1999).

[14] K.M. Watson and J.M. Jauch, Phys. Rev. 75, 1249 (1949).

[15] G.S. Agarwal, Phys. Rev. A 11, 230 (1975), Phys. Rev. A 11, 243 (1975), Phys. Rev. A 11, 253 (1975), Phys. Rev. A 12, 1475 (1975).

[16] B. Huttner, J.J. Baumberg, and S.M. Barnett, Europhys. Lett. 16, 177 (1991).

[17] P.D. Drummond, Phys. Rev. A 42, 6845 (1991).

[18] P.W. Milonni, J. Mod. Opt. 42, 1991 (1995).

[19] D.J. Santos and R. Loudon, Phys. Rev. A 52, 1538 (1995).

[20] P.D. Drummond and M. Hillery, Phys. Rev. A 59, 691 (1999).

[21] B. Huttner and S.M. Barnett, Europhys. Lett. 18, 487 (1992), Phys. Rev. A 46, 4302 (1992).

[22] J.J. Hopfield, Phys. Rev. 112, 1555 (1958).

[23] U. Fano, Phys. Rev. 103, 1202 (1956).

[24] T. Gruner and D.-G. Welsch, Phys. Rev. A 53, 1818 (1996).

[25] T. Gruner and D.-G. Welsch, Third Workshop on Quantum Field Theory under the Influence of External Conditions (Leipzig, 1995) [B.G. Teubner Verlagsgesellschaft, Stuttgart.Leipzig, 1996]. 
[26] Ho Trung Dung, L. Knöll, and D.-G. Welsch, Phys. Rev. A 57, 3931 (1998).

[27] S. Scheel, L. Knöll, and D.-G. Welsch, Phys. Rev. A 58, 700 (1998).

[28] Ho Trung Dung, L. Knöll, and D.-G. Welsch, arXiv:quant-ph/0003138.

[29] W.C. Chew, Waves and Fields in Inhomogeneous Media (IEEE Press, New York,1995). 